\title{
Mediastinite pós-esternotomia longitudinal para cirurgia cardíaca: 10 anos de análise
}

\author{
Valdir Cesarino de SOUZA*, André Ney Menezes FREIRE**, José TAVARES-NETO**
}

(*) Professor-assistente da Faculdade de Medicina de Campina Grande, UFCG, Cirurgião Cardiovascular do ICCP. (**) Professores-adjuntos do Curso de Pós-Graduação em Medicina e Saúde da Faculdade de Medicina da UFBA.

RBCCV 44205-599

\section{Resumo}

Introdução: mediastinites pós-esternotomia para cirurgia cardíaca não são freqüentes $(0,2 \%$ a $5,0 \%)$, porém, quando surgem, se tornam potencialmente graves. Mesmo com o diagnóstico e tratamento precoces, o prognóstico não é bom, sobretudo se houver sepse e outros agravos à saúde associados.

Objetivo: rever a casuística de casos de mediastinite.

Método: foram analisados os prontuários de 2.272 pacientes submetidos à cirurgia cardíaca entre 1991 e 2000. Todas as operações foram realizadas através de esternotomia mediana longitudinal e circulação extracorpórea no Hospital João XXIII / Instituto de Cirurgia Cardiovascular da Paraíba de Campina Grande (Paraíba). Resultados: a mediastinite ocorreu, em média, 10 dias após a cirurgia, num total de 37 $(1,6 \%)$ casos, com taxa de letalidade $21,6 \%(n=8)$. A maioria $(n=19 ; 51,4 \%)$ dos casos foi em pacientes submetidos a revascularização do miocárdio, seguidos pelos procedimentos valvares $(n=13 ; 35,1 \%)$, correções de cardiopatias congênitas $(n=4 ; 10,8 \%)$ e aneurisma de aorta ascendente $(n=1 ; 2,7 \%)$. Vários fatores de risco foram identificados (obesidade, tempo de permanência hospitalar prolongado, diabetes mellitus, tabagismo, reoperações e cirurgias de emergência), especialmente a permanência (por mais de 72 horas no préoperatório) em unidade de terapia intensiva. A cultura do exsudato foi positiva em $35(94,6 \%)$ dos 37 pacientes, sendo o Staphylococcus aureus o patógeno mais observado em 17 $(48,6 \%)$.

Conclusões: a freqüência de mediastinite pós-cirurgias cardíaca, com esternotomia associada, é semelhante à descrita na literatura, não tem diminuído no decorrer dos anos, por isto continua representando um desafio para os cirurgiões e equipes, apesar do arsenal diagnóstico e terapêutico atuais.

Descritores: Mediastinite. Infecção mediastinal. Cirurgia cardíaca, infecção. Infecção cirúrgica.

\section{Abstract}

Introduction: Mediastinitis is a rare though potentially fatal complication. The incidence is reported to be between $0.2 \%$ and $5.0 \%$ and is a major cause of postoperative morbidity. Despite early diagnosis and proper treatment, prognosis is poor because of the chance of mediastinal spread of the infection and poor physical state of these patients.

Objective: To review the casuistic of mediastinites.

Method: The records of all 2,272 patients who underwent cardiac surgical procedures between 1991 and 2000 were reviewed. All operations were performed using longitudinal sternotomy and extracorporeal circulation in João XXIII Hospital / Institute of Cardiovascular Surgery of Paraíba in Campina Grande, Paraíba.

Results: The complication occurred within, on average, 10 days after operation. In total of $37(1.6 \%), 8(21.6 \%)$ deaths occurred. Mediastinitis were reported in $19(51.4 \%)$ cases, in patients submitted to coronary artery bypass grafting, 13 $(35.1 \%)$ in valve diseases, $4(10.8 \%)$ in congenital cardiopathy corrections and $1(2.7 \%)$ in ascending aortic aneurysm. Several risk factors mediastinitis were identified (obesity, prolonged stay in hospital, diabetes mellitus, smoking, reoperation and emergency surgery), especially the prolonged stay in intensive-care unit for more then $\mathbf{7 2}$ hours before cardiac operation. Bacterial cultures of exudates were positive in $\mathbf{3 5}$ $(\mathbf{9 4 . 6 \% )}$ of 37 patients; Staphylococcus aureus was the responsible pathogen in $17(48.6 \%)$ patients.

Conclusion: The incidence of mediastinitis after longitudinal sternotomy following cardiac surgery do not decreased in the last 10 years and represents a surgical challenge, in spite of the progress added to the diagnostic and therapeutic of this pathology.

Descriptors: Mediastinitis. Mediastinal infection. Cardiac surgery, infection. Surgical infection.
Trabalho realizado no Hospital João XXIII e Instituto de Cirurgia Cardiovascular da Paraíba (ICCP), com o apoio da Universidade Federal de Campina Grande (UFCG) - Campina Grande, Paraíba e do Curso de Pós-Graduação em Medicina e Saúde da Universidade Federal da Bahia (UFBA).

Endereço para correspondência: Valdir Cesarino de Souza. Rua Siqueira Campos, 543. Campina Grande, Paraíba, Brasil.

CEP 58108-540 - Tel. 0xx83 3413640 - Fax 0xx83 3414132 e-mail: valdircs@ cardiol.br 


\section{INTRODUÇÃO}

A mediastinite é uma grave complicação infecciosa do pós-operatório de cirurgias abordando o coração e os grandes vasos da base. A prevalência de infecções mediastinais em cirurgia cardíaca, nas quais se usam a via de acesso transesternal, varia de $0,2 \%$ a $5,0 \%{ }^{(1,2)}$. Os riscos de mediastinite aumentam com a presença de algumas afecções pré-existentes ou procedimentos associados, como: desnutrição; diabetes mellitus; revascularização do miocárdio utilizando-se as duas artérias mamárias internas; o uso abusivo de eletrocautério; doença pulmonar obstrutiva crônica (DPOC); uso de corticoterapia prévia; cirurgia prolongada; tabagismo; longo período de internação hospitalar; ventilação mecânica por mais de 72 horas; internação prévia por mais de 72 horas de Unidade de Terapia Intensiva (UTI), entre outros ${ }^{(3,4,5)}$. Não obstante, devido também à falta de um consenso definido, parecendo que cada Instituição tem "seus próprios fatores de risco" associado à mediastinite ${ }^{(6)}$.

Devido às complicações decorrentes a mediastinite pósoperatória (instabilidade esternal insuficiência respiratória, sepse, entre outras), tem-se indicado procedimentos cirúrgicos menos invasivos à abordagem mediastinal, utilizando-se minitoracotomias com ou sem o auxílio de videotoracoscopia ${ }^{(7,8,9)}$. Assim, têm sido alcançados baixíssimos índices de morbidade, porém essas e outras técnicas menos invasivas têm suas limitações ${ }^{(10)}$. Também, apesar do aperfeiçoamento de inúmeras alternativas cirúrgicas para debelar o processo infeccioso ${ }^{(11-19)}$, persiste o risco de mediastinite e que pode ser a causa de óbito em até $47 \%$ dos casos ${ }^{(20)}$.

Os autores ${ }^{(8-10,21,22)}$ vêem demonstrado concordâncias quanto à diminuição da morbi-mortalidade entre os vários tipos de acesso mediastinal, menos invasivos, e a conseqüente recuperação mais precoce, dos pacientes submetidos aos diversos procedimentos cirúrgicos cardiológicos. Não obstante, no Brasil, especialmente na região Nordeste, existem poucos estudos de prevalência sobre a mediastinite, e por isto o interesse de rever os casos de cirurgia cardíaca, com acesso transesternal, visando também a descrição de indicadores demográficos, clínicoepidemiológicos e etiológicos, observados em casos de Campina Grande (PB).

\section{MÉTODO}

O estudo transversal retrospectivo foi realizado no Hospital João XXIII/Instituto de Cirurgia Cardiovascular da Paraíba, ambos localizados em Campina Grande (Paraíba), com os pacientes submetidos a cirurgias cardíacas através de esternotomia mediana transesternal entre janeiro de 1991 e dezembro de 2000. A localização dos prontuários dos pacientes foi realizada pelo registro de intercorrências e do arquivo catalográfico dos indivíduos operados naquele intervalo de 10 anos. Após isto, todos os casos foram incluídos no banco de dados do software SPSS (Statistical Package for Social Sciences) versão 9.0. Foram revistos os casos com diagnóstico de mediastinite (pacientes que apresentavam febre, dores e exsudatos localizados na ferida operatória, mobilidade esternal, leucocitose e instabilidade clínica, entre outros) ${ }^{(5,6,13,20)}$. Foram excluídos aqueles prontuários que apresentavam esses critérios de inclusão, porém, com relatórios médicos incompletos, falta de exames complementares e/ou quando o paciente abandonou o tratamento.

O diagnóstico de mediastinite foi realizado por meio do acompanhamento clínico no pós-operatório, observando os seguintes indicadores: dor e hiperemia ao nível da ferida cirúrgica; leucocitose; instabilidade esternal; exsudato local; piora das condições clínicas e derrames pericárdicos e pleurais; entre outros. Também, os exames complementares (radiografia de tórax e ecocardiografia), auxiliaram no diagnóstico mais precoce da presença de líquidos mediastinais, bem como exames microbiológicos (hemoculturas, cultura de pontas de catéteres e de exsudato), na investigação da etiologia de processo infeccioso. Por não haver realizado tomografia computadorizada do tórax em todos os pacientes, este exame não foi incluído entre os critérios de diagnóstico. A ressonância magnética não foi executada em nenhum paciente, por ser indisponível no serviço.

Quando diagnosticada a mediastinite, foram utilizados aqueles e outros meios diagnósticos auxiliares para caracterizar o caso ou acompanhar a evolução clínica do mesmo: hemograma; eletrólitos; uréia; creatinina; exames gasométricos; cultura de exsudatos e exame radiológico simples (radiografia do tórax na posição ântero-posterior), para avaliar se havia aumento significativo da silhueta cardíaca. Diante disto, foram indicados exames mais específicos como a ecocardiografia transtorácica, para avaliação da extensão e do volume do exsudato mediastinal.

\section{RESULTADOS}

Durante o período de 10 anos (1991 a 2000), um total de 2.272 pacientes foi submetido a intervenções cirúrgicas com abordagem cardíaca, por meio da esternotomia mediana. Destes, $1.017(44,8 \%)$ sofreram revascularização do miocárdio; 763 (33,6\%) correções de defeitos valvares; 396 $(17,4 \%)$ submetidos à correção de cardiopatias congênitas e $96(4,2 \%)$ a tratamento de diversas patologias. Neste grupo de estudo, 41 (1,8\%) pacientes desenvolveram mediastinite, porém, aplicando-se os critérios de seleção do estudo, foram excluídos 4 pacientes (por dados incompletos) e assim foram selecionados $37(1,6 \%)$ entre aqueles 2.272 pacientes: 19 do grupo coronariano $(1,9 \%) ; 13(1,7 \%)$ do grupo de valvopatias; $4(1,0 \%)$ dos pacientes de cardiopatias congênitas e $1(1,0 \%)$ daqueles submetidos ao tratamento de outras patologias cardíacas. Essas proporções, analisadas (qui-quadrado) em tabelas $2 \times 2$ (e.g. grupo coronariano vs. valvopatias, etc.), foram estatisticamente 
semelhantes ( $p>0,26$ ), bem como ao comparar ( $p>0,95$; com correção de Yates) a proporção dos três primeiros grupos (36/2176) versus os do grupo com outras patologias (1/96).

Houve predomínio $(\mathrm{n}=20 ; 54,0 \%)$ de pacientes do gênero feminino. A média geral de idade foi de 51,9 $(+21,1)$ anos, com limites de 1,2 (três eram crianças, com menos de 4 anos) a 82,6 anos, sendo no gênero masculino de $57,1(+23,1)$ anos e no feminino de 47,5 $(+18,8)$. Os principais fatores de risco pré-operatórios foram: obesidade $(n=15 ; 40,5 \%)$; internações prévias em UTI por mais de 72 horas $(n=15$; $40,5 \%)$; tabagismo $(\mathrm{n}=14 ; 37,8 \%)$; diabetes mellitus $(\mathrm{n}=5$; $13,5 \%)$; reoperações $(n=4 ; 10,8 \%)$ e cirurgias de urgência $(\mathrm{n}=1 ; 2,7 \%) . \operatorname{Em} 7(18,9 \%)$ pacientes não foram observados fatores de risco prévios.

A queixa inicial mais freqüente $(91,9 \%)$ foi a dor retroesternal, habitualmente de forte intensidade. Foram classificadas diversas apresentações de mediastinite: aguda e crônica, segmentar e difusa, associada ou não à instabilidade esternal. O tempo de aparecimento da mediastinite aguda $(n=29)$ variou entre 4 e 12 dias, com média de 5,8 $(+2,0)$ dias e da mediastinite crônica $(n=8)$ entre 27 e 154 dias, sendo a média de 57,4 (+ 41,7) dias. Alguns pacientes $(n=8)$ desenvolveram infecção em apenas uma determinada região da ferida operatória (mediastinite segmentar). Em 29 (78,4\%) pacientes, a mediastinite foi também difusa. Do total $(\mathrm{n}=37)$ de pacientes, a maioria $(\mathrm{n}=19$; $51,4 \%$ ) evoluiu com instabilidade esternal, todos da forma aguda-difusa. Dos $29(78,4 \%)$ pacientes que desenvolveram a infecção aguda, seis $(16,2 \%)$ eram de forma restrita a determinado segmento do mediastino, sem mobilidade do esterno e $23(62,2 \%)$ evidenciavam o mediastino difusamente comprometido, sendo que $19(51,4 \%)$ apresentavam mobilidade esternal e $4(10,8 \%)$ não. Entre os $8(21,6 \%)$ pacientes que evoluíram para a mediastinite crônica, nenhum apresentava mobilidade do esterno, dois $(5,4 \%)$ tinham a forma segmentar da doença e $6(16,2 \%)$ a difusa.

Todos os indivíduos desta série foram submetidos à circulação extracorpórea com tempo médio de 72,0 (+ 17,0) minutos e limites de 36 a 135 minutos. Entre os 37 casos estudados, a maioria $(56,8 \% ; \mathrm{n}=21)$ saiu de alta hospitalar e os demais casos ou desenvolveram mediastinite crônica $(n=8 ; 21,6 \%)$ ou foram a óbito $(n=8 ; 21,6 \%)$, sendo o tempo médio de circulação extracorpórea nesses grupos, respectivamente, de 69,2 (+ 14,2), 77,0 (+21,6) e 71,1 (+26,3) minutos.

O Staphylococcus aureus foi o microorganismo mais freqüentemente isolado na ferida operatória em 17 casos (46,0\%), seguido da Pseudomonas aeruginosa em 8 casos (21,6\%); Escherichia coli em 4 casos (10,8\%); Klebsiella sp. em 3 casos $(8,1 \%)$; S. epidermidis em outros 3 casos $(8,1 \%)$; e em 2 casos $(5,4 \%)$ a cultura bacteriana foi negativa, provavelmente devido ao uso prévio de antibiótico. Todos os 3 casos com isolamento de $S$. epidermidis desenvolveram mediastinite crônica.

A Tabela 1 mostra a distribuição do tipo de saída hospitalar, segundo o resultado da cultura bacteriológica.
Chama a atenção, apesar do pequeno número de casos, a freqüência de óbitos $(75,0 \%)$ entre aqueles (3/4) com E. coli isolada no exsudato.

Tabela 1. Freqüência do tipo de saída hospitalar dos casos de mediastinites, segundo o agente etiológico.

\begin{tabular}{lcccc}
\hline \multirow{2}{*}{ BACTÉRIA ISOLADA } & \multicolumn{4}{c}{ RESULTADO FINAL n (\%) } \\
\cline { 2 - 5 } & Curado & $\begin{array}{c}\text { curado } \\
\text { (crônico) }\end{array}$ & Óbito & TOTAL \\
\hline S. aureus & $12(70,6)$ & $4(23,5)$ & $1(5,9)$ & 17 \\
P. aeruginosa & $5(62,5)$ & - & $3(37,5)$ & 8 \\
E. coli & $1(25,0)$ & - & $3(75,0)$ & 4 \\
Klebsiella sp. & $2(66,7)$ & $1(33,3)$ & - & 3 \\
S. epidermidis & - & $3(100)$ & - & 3 \\
Cultura negativa & $1(50,0)$ & - & $1(50,0)$ & 2 \\
TOTAL & $21(56,8)$ & $8(21,6)$ & $8(21,6)$ & 37 \\
\hline
\end{tabular}

Na Tabela 2 estão descritos os procedimentos cirúrgicos, usados no tratamento dos casos de mediastinite, sendo que a maioria $(46,0 \%)$ foi por meio de debridamento com irrigação contínua do mediastino com solução iodada.

Tabela 2. Procedimentos cirúrgicos realizados no tratamento da mediastinite pós-esternotomia mediana em cirurgia cardíaca, em Campina Grande, $P B$.

\begin{tabular}{lc}
\hline P R O C E D I M E N T O S & n (\%) \\
\hline Debridamento com drenagem simples do mediastino & $8(21,6)$ \\
$\begin{array}{l}\text { Debridamento com irrigação contínua do mediastino } \\
\text { Debridamento com retalho do músculo grande peitoral }\end{array}$ & $17(46,0)$ \\
$\begin{array}{l}\text { Debridamento com irrigação contínua do mediastino + retalho do músculo grande } \\
\text { peitoral }\end{array}$ & $1(2,7)$ \\
$\begin{array}{l}\text { Debridamento com drenagem contínua + retalho do músculo grande peitoral + } \\
\text { enxerto ósseo }\end{array}$ & $1(2,7)$ \\
$\begin{array}{l}\text { Debridamento com drenagem simples do mediastino + retalho do músculo reto } \\
\text { abdominal }\end{array}$ & $1(2,7)$ \\
$\begin{array}{l}\text { Debridamento com irrigação contínua do mediastino + retalho do grande epíplon } \\
\text { Debridamento com irrigação contínua do mediastino + enxerto ósseo + retalho do } \\
\text { grande epíplon }\end{array}$ & $2(5,4)$ \\
\hline
\end{tabular}

As principais complicações e estados associados observados nos casos de mediastinite foram: insuficiência respiratória $(n=16)$; acidente vascular cerebral $(n=6$, todos isquêmicos, comprovados através de tomografia computadorizada); choque cardiogênico $(n=5)$; insuficiência renal aguda ( $\mathrm{n}=11$, sendo 4 submetidos à hemodiálise); 8 pacientes apresentaram sangramento acentuado no pósoperatório imediato (todos politransfundidos); 14 (37,8\%) evoluíram com quadro de infecção pulmonar e $6(16,2 \%)$ desenvolveram quadro séptico com falência de múltiplos órgãos.

Do total de casos falecidos $(\mathrm{n}=8)$, seis apresentaram, na evolução clínica, falência de múltiplos órgãos (todos também com tempo prolongado de ventilação mecânica) e outros 2 pacientes com insuficiência renal aguda.

\section{COMENTÁRIOS}

O diagnóstico precoce da mediastinite é fundamental para o bom prognóstico. A febre persistente, a presença de supurações e/ou mobilidade esternal, a dor localizada ao nível da ferida operatória, a queda do estado geral do paciente, a leucocitose, entre outros indicadores, são fortes 
indícios daquela complicação.

A taxa de infecção em cirurgias gerais, limpas, no Hospital João XXIII/Instituto de Cirurgia Cardiovascular da Paraíba é de $2,1 \%$, sendo na série estudada de $1,6 \%$ e que é semelhante à descrita na literatura ${ }^{(2,6)}$. Durante a última década do século passado, as prevalências de mediastinite em vários serviços, apesar das novas técnicas cirúrgicas e os antibióticos mais potentes, sugerem a necessidade da revisão sistemática dos procedimentos e condutas, na busca do aprimoramento ou correção de alguns destes.

Pacientes internados, previamente, por mais de 3 dias em UTI, foram os mais acometidos de mediastinite devido, provavelmente, à colonização prévia da pele e mucosas com bactérias do âmbito hospitalar ${ }^{(3,5)}$, apesar das medidas terapêuticas preventivas, recomendadas na literatura, realizadas antes do ato operatório ${ }^{(6)}$ como: o exame clínico, buscando focos infecciosos; realização de banhos com soluções bactericidas; uso profilático de antibióticos (cefalotina e gentamicina) uma hora antes do ato operatório e durante a própria intervenção cirúrgica (a cada duas horas).

Os fatores de risco, descritos na literatura ${ }^{(3,5,6)}$, foram observados em $30(81,1 \%)$ pacientes, entre os quais os mais freqüentes, além da internação prévia em UTI, foram a obesidade e o tabagismo.

Várias condutas terapêuticas foram preconizadas para debelar o processo infeccioso. A primeira delas foi à reavaliação da antibioticoterapia logo após o resultado do estudo bacteriológico. Adicionalmente, foram realizados diversos procedimentos operatórios, conforme o caso, como o debridamento e lavagem da cavidade mediastinal com drenagem simples.

O mesmo procedimento, com irrigação contínua com solução bactericida a base de iodo (Povidine ${ }^{\circledR}$ ), foi realizado na maioria $(\mathrm{n}=27 ; 73,0 \%)$ dos pacientes, e por períodos determinados (aproximadamente 15 dias) e a negativação bacteriológica do exsudato, foi obtida em 21 casos. Na literatura, tanto SHUMAKER et al. (1963) como VERKKALA et al. (1986) e BJERNO et al. (1990), já relatavam o grande sucesso na negativação de culturas de exsudatos mediastinais pós-irrigação contínua do mediastino com soluções bactericidas por um período médio de 12,7 dias após a detecção da infecção.

Após aqueles procedimentos cirúrgicos, quando não eram passíveis de solução definitiva, foram adotadas condutas mais agressivas como a interposição mediastinal de retalho do grande peitoral e do reto abdominal, conforme preconizaram MAJURE et al. (1986) e COLEN et al. (1989), descrevendo-o como sendo o melhor procedimento e a primeira escolha de tratamento quando há perda significativa de substâncias na porção inferior do esterno.

Além destas técnicas, foram instituídos outros procedimentos, como a laparotomia exploradora com rotação do grande epíplon em direção ao mediastino anterior, via transxifoidea, ocupando o espaço outrora preenchido pela fáscia endotorácica, técnica essa difundida por LEE et al.
(1976) e SAMPAIO et al. (2000).

Em 3 pacientes com osteomielite do esterno foram realizadas condutas ainda mais agressivas, como a remoção total do esterno e cartilagens condrais com interposição de enxerto ósseo (crista ilíaca), além de interposição de retalhos do grande epíplon ( 2 casos) e musculares ( 1 caso) conforme preconizaram SAMPAIO et al. (2000) e NAHAI et al. (1989), respectivamente.

Diversas medidas preventivas ${ }^{(6)}$ contra a mediastinite foram descritas na literatura, dentre elas: a rigorosa avaliação pré-operatória do paciente em busca de focos infecciosos prévios (amigdalites, faringites, periodontite, piodermites, entre outros); em cirurgias eletivas, a interrupção do tabagismo por cerca de 15 dias antes de cirurgia; evitar a dissecção das duas artérias torácicas internas (principalmente em pessoas idosas e diabéticas); reduzir, ao máximo, o tempo de internação hospitalar principalmente antes da cirurgia, evitando assim a colonização do paciente por microorganismos altamente selecionados do ambiente hospitalar; fazer assepsia rigorosa do paciente com soluções anti-sépticas; usar o mínimo possível o eletrocautério na diérese; manipular cuidadosamente os tecidos; evitar, ao máximo, o trauma cirúrgico do esterno; fazer hemostasia rigorosa; orientar, periodicamente, os integrantes da equipe cirúrgica e anestésica, como também avaliar os equipamentos utilizados no centro cirúrgico ou no de tratamento intensivo; manipular adequadamente os drenos, os catéteres e os curativos operatórios, entre outras.

\section{CONCLUSÃO}

A mediastinite, complicação cirúrgica bastante temida pelas equipes de cirurgia cardíaca, continua sendo de controle difícil. O diagnóstico precoce e a intervenção terapêutica, como a ressecção total ou parcial do tecido infectado, são fundamentais para a boa recuperação do paciente. $\mathrm{O}$ aparecimento de novas possibilidades intervencionistas ${ }^{(7-11)}$ deverá levar à melhoria dos resultados, principalmente na prevenção de mediastinite pós-operatória ou de suas recorrências. A diversidade de situações, o estado de saúde prévio do paciente, a alta morbimortalidade dessa complicação, obrigam os pesquisadores de todo o mundo a continuar a busca de novos métodos diagnósticos e terapêuticos, cada vez mais eficazes para debelar essa tão temida complicação, bem como a revisão sistemática dos procedimentos atuais visando o aperfeiçoamento das técnicas e condutas utilizadas.

Contudo, para se evitar a mediastinite e suas graves conseqüências médico-sócio-econômicas há necessidade, sobretudo, de adoção de algumas medidas: instituir rigorosas condutas de prevenção pré, per e pós-cirúrgicas e, principalmente, realizar precocemente a terapia aos primeiros sinais de infecção, mas antes buscando, por todos os meios, pesquisar o agente etiológico. 


\section{REFERÊNCIAS BIBLIOGRÁFICAS}

1. Berg HF, Brands WG, van Geldrop TR, KluytmansVanden Bergh FQ, Kluytmans JA - Comparison between closed drainage techniques for the treatment of postoperative mediastinitis. Ann Thorac Surg 2000; 70: 924-9.

2. Sarr MG, Gott VL, Townsend TR - Mediastinal infection after cardiac surgery. Ann Thorac Surg 1984; 38: 415-23.

3. Farinas MC, Gald Peralta F, Bernal JM, Rabasa JM, Revuelta JM, Gonzalez-Macias J - Suppurative mediastinitis after open-heart surgery: a case-control study covering a seven-year period in Santander, Spain. Clin Infect Dis 1995; 20: 272-9.

4. Braxton JH, Marrin CA, McGrath PD et al. - Mediastinitis and long-term survival after coronary artery bypass graft surgery. Ann Thorac Surg 2000; 70: 2,004-7.

5. Tarelli G, Maugeri R, Pedretti R, Grossi C, Ornaghi D, Sala A - The use of bilateral mammary artery in miocardial revascularization. The risk factors emergent from a multivariate analysis conducted on 474 patients. G. Ital Cardiol 1998; 28: 1,230-7.

6. Sampaio DT, Alves JCR, Silva AF et al. - Mediastinitis after cardiac surgery: treatment using omental flap. Braz Jour Cardiov Surg 2000; 15: 23-31.

7. Cosgrove DM, Sabik JF - Minimally invasive approach for aortic valve operation. Ann Thorac Surg 1996; 62: 596-7.

8. Marcial MB, Tanamati C, Jatene MB, Atik E, Jatene AD - Transxiphoid approach without median sternotomy for the repair of atrial septal defects. Ann Thorac Surg 1998; 65:771-4.

9. Lazzara R, Kidwell FE - Right parasternal incision: A uniform minimally invasive approach for valve operations. Ann Thorac Surg 1998; 65:271-2.

10. Calafiore AM, Giamarco GD, Teodori Get al. - Left anterior descending coronary artery grafting via left anterior small thoracotomy without cardiopulmonary bypass. Ann Thorac Surg 1996; 61: 1,658-63.

11. Acuff TE, Landreneau RJ, Griffith BP, Mack MJ Minimally invasive coronary artery bypass grafting. Ann Thorac Surg 1996; 61: 135-7.
12. Shumacker Jr HB, Mandelbaum I - Continuous antibiotic irrigation in treatment of infection. Arch Surg 1963; 86: 54-7.

13. Bjerno T, Arendrup HC, Alstrup P - Mediastinal infection following open-heart surgery. Uges Laeg 1990; 152: 3,699-702.

14. Verkkala K - Occurrence of and microbiological findings in postoperative infections following open-heart surgery. Effect on mortality and hospital stay. Ann Clin Res 1987; 19: 170-7.

15. Lee Jr AB, Schimert G, Shaktin S, Seigel JH - Total excision of the sternum and thoracic pedicle transposition of the greater omentum: useful stratagems in managing severe mediastinal infection following open-heart surgery. Surg 1976; 80:433-6.

16. Sampaio LCN - Estudo comparativo entre a miniesternotomia em L invertido e a esternotomia longitudinal total para a realização de atrioseptoplastia. [Dissertação. Mestrado] Salvador: Faculdade de Medicina da Universidade Federal da Bahia, 2000. 74p.

17. Nahai F, Rand RP, Hester TR, Bostwick J, Jurkiewicz MJ - Primary treatment of the infected sternotomy wound with muscle flaps: a review of 211 consecutive cases. Plast Reconstr Surg 1989; 84: 434-41.

18. Majure JA, Albin RE, O'Donnell RS, Arganese TJ Reconstruction of the infected median sternotomy wound. Ann Thorac Surg 1986; 42: 9-12.

19. Colen LB, Huntsman WT, Morain WD - The integrated approach to suppurative mediastinitis: rewiring the sternum over transposed omentum. Plast Reconstr Surg 1989; 84: 936-41.

20. El Oakley RM, Wright JE - Postoperative mediastinitis: classification and management. Ann Thorac Surg 1996; 61: 1,030-6.

21.Brutel R, Brom GH, Brom AG - Horizontal submammary skin incision for median sternotomy. Ann Thorac Surg 1981;32: 101-4.

22. Nair RU, Sharpe DA - Minimally invasive reversed Z sternotomy for aortic valve replacement. Ann Thorac Surg 1998; 65: 165-6. 